\title{
Cohort study on living arrangements of older men and women and risk for basic activities of daily living disability: findings from the AGES project
}

\author{
Tami Saito $^{1 *}$ (D) Chiyoe Murata ${ }^{1}$, Jun Aida ${ }^{2}$ and Katsunori Kondo 3,4
}

\begin{abstract}
Background: Living arrangements of older adults have changed worldwide with increasing solitary and non-spouse households, which could affect social care systems. However, the relationship between these households and disability onset has remained unclear. We examined the relationship between living arrangements and the onset of basic activities of daily living disability in older adults, with a focus on gender differences and cohabitation status of those without a spouse.

Methods: Data from 6600 men and 6868 women aged 65 years or older without disability were obtained from the Aichi Gerontological Evaluation Study Project in Japan. Onset of disability was followed for 9.4 years. Disability was assessed based on Long-term Care Insurance System registration. A hierarchical Cox proportional hazards model was conducted to examine the risk of living alone and living only with non-spousal cohabitants compared to those living with spouses.

Results: Men living only with non-spousal cohabitants and those living alone were significantly more likely to develop disability after controlling for health and other covariates (hazard ratio $=1.38$ and 1.45, respectively), while a significant difference was found only for women living alone (hazard ratio $=1.19$ ). The risk of living with non-spousal cohabitants was marginally stronger in men, indicated by the interaction effect model $(p=.08)$. A series of hierarchical analyses showed that social support exchange explained $24.4 \%$ and $15.8 \%$ of the excess risk of disability onset in men living alone and those living only with non-spousal cohabitants, respectively. A subsequent analysis also showed that support provision by older adults more greatly explained such excess risk than receiving support from others.

Conclusions: Older men without spouses were more likely to develop disability onset regardless of cohabitants. Health professionals should consider programs that enhance social support exchange, particularly support provision by older adults who are at risk of disability.
\end{abstract}

Keywords: Basic activities of daily living, Living arrangements, Longitudinal study, Japanese

\footnotetext{
* Correspondence: t-saito@ncgg.go.jp

'Department of Social Science, National Center for Geriatrics and

Gerontology, 7-430 Morioka-cho, Obu, Aichi 474-8511, Japan

Full list of author information is available at the end of the article
} 


\section{Background}

Prevention of functional disability among older individuals and related costs to the healthcare system are a pressing issue in aging societies $[1,2]$. In countries with the most progressed aging populations, such as Japan, the proportion of older people is expected to reach $40 \%$ in 2050 [3].

Living arrangements of older adults have changed dramatically over time [4]. The proportion of older adults living alone is increasing worldwide [5], which could affect care provision by the community [6] and necessitates more detailed focus on their health problems and needs. In addition, the proportion of older adults without spouses living in the household could increase in the future. For instance, in the United States, about one third of baby boomers were unmarried [7]. Such changes in living arrangements imply the growing importance of examining the role of non-spousal networks in older adults' health.

Many longitudinal studies have reported marital advantage regarding mortality and morbidity [8-12]; in contrast, relatively few studies have examined the relationship between functional disability and living with non-spousal cohabitants [11-17]. The presence of nonspousal family members of the unmarried could compensate for the lack of marital protection for older adults [18], while solitary living could promote independence among older adults [19]. Previous longitudinal studies have shown that older adults who lived only with non-spousal family members were more likely to develop functional decline compared to those who lived with spouses $[12,14,15]$ or those who lived alone [11, 13, 16, 17]. Regarding those living alone, there have been inconsistent findings of comparable risk [11, 15, 16], excess risk [20], or less risk [14] compared to older adults living with a spouse.

Based on these findings, several points are worth further consideration. First, gender differences could exist in the association between living arrangements and onset of disability. Studies have shown more benefits for men living with a spouse compared to women for mortality $[8,21]$ and morbidity [10]. The association between living with non-spousal cohabitants and health could also differ by gender, since intimate relationships were more likely to be limited to a wife in older men, while older women relied on more diverse individuals such as their children [22]. However, few longitudinal studies have examined gender differences in the relationship between living arrangements and disability $[11,20]$.

Second, several factors could mediate the association between living arrangements and onset of disability. In studies on marital status and health, "marital protection [8]," referring to benefits obtained because of marriage, such as economic resources, social resources such as social support networks $[7,8,10]$, and control over health-related behaviors [10], affects health in older adults $[8,13]$. This has been observed even when considering the association of "marital selection," a precursor to marital status [8]. Also, mental disorders in middle-aged adults differed by types of living arrangement, partially due to differential social support and unhealthy behaviors [10]. However, it remains unclear which factors mediate the association between living arrangements and onset of disability.

Third, needs-driven cohabitation among older adults, meaning those needing support tend to live with family members, particularly with their adult children [23], could confound the findings [17]. In Japan, living arrangements of older adults are relatively stable [24] because of low rates of needs-driven cohabitation [25] and remarriage [26]. Takagi and colleagues examined types of cohabitation with children and indicated that among older adults residing with their children, nearly $80 \%$ resided with their children their entire life [25]. This suggests that most older adults who reside with their children started living with them before the onset of health decline. Thus, reverse causation is less likely to be an issue as compared to Western countries, where needs-driven cohabitation is dominant [23].

The aim of this study was to examine the relationship between living arrangements and the onset of BADL disability among older adults with a focus on gender differences and cohabitation status in those living without a spouse. Following this, we also examined how social support and health-related behaviors mediated these associations.

\section{Methods}

\section{Participants}

Data were obtained from the Aichi Gerontological Evaluation Study (AGES) cohort dataset, which was part of the Japan Gerontological Evaluation Study (JAGES) project. A self-administered questionnaire survey was conducted in October 2003 with 33,152 people aged 65 years or older who were not eligible to utilize Longterm Care Insurance (LTCI) system services and who were selected through simple randomization (6 municipalities) or complete enumeration (4 small-scale municipalities) in Aichi Prefecture, Central Japan (response rate $=52.1 \%$ ). The details of the survey were shown elsewhere $[1,27]$.

Survey data from 15,313 respondents who provided information for identification by the LTCI system were linked to the LTCI records dataset for a follow-up period of 3436 days (9.4 years) from November 1, 2003. We excluded data from 401 respondents who had qualified for LTCI benefits with a level 2 or higher rating by October 31, 2004, 332 who did not apply for LTCI benefits despite having BADL limitations, and 806 who did not provide information on the BADL items, to avoid 
the problem of reverse causation. We also excluded data for 306 cases for which information on living arrangements was not provided. Data for a total of 13,468 respondents (6600 men and 6868 women) were finally included in the analysis.

\section{Measurements \\ Outcome}

We collected information on the onset of BADL decline from the LTCI records administrated by municipalities. The LTCI system classifies frail older adults into seven levels (Support need levels 1 and 2 and Care need levels 1 to 5; a larger number indicates a more severe level) using a nationally standardized and validated algorithm. The levels are solely determined according to older adults' physical and mental care needs, regardless of informal care provided to the recipients [28] and assessed by both computer-based and home-visit interviews by a trained healthcare professional and examination by a primary physician [29]. In the computer-based assessment, time required for care is calculated according to nine categories of care needs such as five domains of BADL care (bathing, eating, toileting, dressing, and transferring), assistance with instrumental activities of daily living (IADL), behavioral problems, rehabilitation, and medical services [28]. In our study, BADL decline was defined as a new registration within the LTCI records with a care-needs level of 2 or above, which requires $50 \mathrm{~min}$ of care or longer per day and nearly corresponds to the need for any type of BADL care [29]. More detailed information was shown in another study [28].

\section{Living Arrangements}

Japanese families have been traditionally based on the "stem family" system, indicating cohabitation of parent(s) and one of their children (typically, the eldest son and his wife) [30]. Although a recent trend shows an increase in solitary households, the majority of older adults live with their spouse and/or children [31]. Since our focus was on examining the risk of cohabitation status of older adults without spouses, we created a living arrangement variable with three categories: living with a spouse, living without a spouse but with at least one non-spousal cohabitant, and living alone. Previous studies in Asian countries [11,15] showed no significant differences based on the presence of children among those who lived with a spouse, suggesting that living with a spouse could comprise one category regardless of the presence of other family members. Regarding older adults living only with non-spousal cohabitants, about 90\% lived with their children, grandchildren, or other blood relatives in this study. Although it is possible that older adults living only with in-law families differ psychosocially from those living with blood relatives, our preparatory analysis showed almost no significant differences between these groups, except for education and income. Therefore, we regarded them as a group. Additionally, a total of 20 cases among 13,468 respondents lived only with non-relatives. Those people were included in the category of older adults living only with non-spousal cohabitants.

\section{Mediators}

We regarded health-related behaviors and social support as possible mediators in the relationship between living arrangements and onset of BADL decline according to the context of marital protection $[7,8,10]$. In terms of health-related behaviors, we assessed smoking habits (none vs. past/current), alcohol consumption (none vs. yes), and body mass index, which was calculated using the respondent's self-rated height and weight, and categorized into "less than $18.5 \mathrm{~kg} / \mathrm{m}^{2}$," "18.5-24.9 kg/m²," and " $25.0 \mathrm{~kg} / \mathrm{m}^{2}$ or over." In addition, daily walking time (less than $30 \mathrm{~min}$ vs. $30 \mathrm{~min}$ or longer) was assessed as a potential physical activity mediator, since walking was one of the most popular physical activities in Japanese older adults [32] and known as a predictor of physical function [33]. Regarding social support, we assessed emotional support received ("Do you have someone to listen to your concerns or complaints?"), emotional support provided ("Do you listen to someone's concerns and complaints?"), instrumental support received ("Do you have someone who looks after you when you are sick and confined to a bed for a few days?"), and instrumental support provided ("Do you look after someone when he/she is sick and confined to a bed for a few days?"). Each support variable had "yes" or "no" response options.

\section{Covariates}

Covariates in this study were selected according to previous studies on risk factors for functional decline [34]: age, education, income, and baseline health status as potential precursors of living arrangements of older adults [23]. In terms of health variables, we assessed self-rated health, presence of illness, depression, IADL, and subjective cognitive complaints (SCC). Self-rated health was assessed using one question: "How do you rate your health?" Response options ranged from excellent to poor, and were dichotomized into two categories (excellent/ good vs. fair/poor). Presence of illness was assessed in terms of whether participants had at least one illness such as cancer, heart disease, stroke, hypertension, diabetes, obesity, hyperlipidemia, osteoporosis, arthritis, trauma, respiratory illness, gastrointestinal illness, liver illness, mental illness, dysphagia, visual/hearing impairment, or incontinence. Depression was assessed with a 15-item Japanese version of the Geriatric Depression Scale [35]. Scores on the scale were categorized into 
three groups: "no depression (0-4 points)," "depressive tendency (5-9)," and "depression (10 and above)." We assessed IADL using a five-item subscale from the Tokyo Metropolitan Institute of Gerontology Higher Competence Scale. The scale was developed based on Lawton's model of competence [36] and its validity and reliability were confirmed [37]. We dichotomized respondents based on their having difficulty with at least one item, for instance, shopping for daily necessities. We assessed SCC using one item asking respondents if they often perceived themselves to be disoriented. Equivalized household income (low, middle, or high), years of education (less than 10 years, or 10 years or more), age in years, and gender (male or female) were also assessed. All mediators and covariates included a missing category, except for age and gender.

\section{Analysis}

All analyses were conducted by gender because we hypothesized that the relationships between living arrangements and BADL disability would differ by gender. After presenting descriptive statistics and differences for each variable among the three living arrangement categories, we examined the relationship between living arrangements and the onset of BADL disability using a Cox proportional hazards model by entering the group of covariates and mediators hierarchically. First, only age was controlled (Model 1). Second, household income, education, and health variables were added to Model 1 (Model 2) to examine the relationship of living arrangements, excluding differences based on the precursors. Additionally, we conducted a series of sub-analyses that examined the differences between the two non-spouse household groups in men and women. Further, to examine gender differences in the relationship between living arrangements and onset of BADL disability, we entered the cross-product terms of living with cohabitants or living alone by gender using the whole sample.

The next three models were employed to examine the influence of mediators on the relationship between living arrangements and BADL disability onset. We added each group of health-related behavior variables and social support variables to Model 2 (Models 3 and 4). Finally, all covariates were entered with living arrangements in the analytical model (Model 5). As a sensitivity analysis, we conducted mediation analysis for Models 3 and 4 to estimate mediation effects of health-related behavior variables (Model 3) and social support variables (Model 4). For this analysis, we applied logistic regression models instead of Cox proportional hazards models due to the limitation of the software program. Stata command "ldecomp" was used for the mediation analysis [38].

Although missing cases for each covariate were modest (24.8\% at the most), $41.1 \%$ of the analyzed respondent data had a missing score for at least one covariate. Therefore, in those multivariate models, we performed a multiple imputation by chained equations under the assumption of missing at random. We generated 20 datasets, analyzed them separately, and pooled the estimates and standardized errors applying Rubin's rules [39]. For mediation analysis, complete case analysis was applied. We regarded respondents who died or who were lost to follow-up due to relocation before the onset of BADL disability as censored cases. All analyses were conducted using IBM SPSS $22.0 \mathrm{~J}$ for Windows (IBM Japan Ltd., Tokyo, Japan) and STATA SE version 14.1 (Stata Corp., College Station, TX, USA). Statistical significance was set at $p<.05$.

\section{Results}

Tables 1 and 2 show the respondents' characteristics by living arrangement status for men and women. For men, $85.5 \%$ lived with a spouse, $10.3 \%$ lived only with nonspousal cohabitants, and $4.2 \%$ lived alone; fewer women lived with a spouse (54.1\%). Those living only with nonspousal cohabitants were older than those living with spouses for both men and women. Men living alone were more likely to have a depressive tendency or depression than those living only with non-spousal cohabitants or those living with a spouse. In contrast, IADL difficulty was less likely among men living alone compared to those living with non-spousal cohabitants or those living with a spouse. Men living alone were less likely to receive and provide emotional and instrumental support than those living with a spouse. For women, those living alone were less likely to receive or provide instrumental support than those with a spouse.

Table 3 shows the incidence rate of the onset of BADL disability during the 9.4-year follow-up period in each category of the explanatory variables. A total of 1108 men and 1248 women showed new onset of disability, with incidence rates per 1000 person-years being 21.4 and 22.3, respectively, for men and women. In men, the incidence rates were 19.4, 35.6, and 32.4, in those with a spouse, living only with non-spousal cohabitants, and living alone, respectively. In women, the rates were 15.0, 34.0 , and 27.8, in those who lived with a spouse, those without a spouse and with non-spousal cohabitants, and those living alone, respectively. The incidence rate of onset of BADL disability in those aged 85 and older was more than 10 times higher than those aged 65 to 69 in both men and women.

Table 4 shows the relationship of living without a spouse (with non-spousal cohabitants or without any cohabitants) for men and women. In Model 1, adjusting for age, both non-spouse household groups were significantly related to the onset of BADL disability in men (hazard ratio $[\mathrm{HR}]=1.39$ for men only with non-spousal 
Table 1 Respondent characteristics by living arrangements for men ( $N=6600)$

\begin{tabular}{|c|c|c|c|c|c|}
\hline \multirow[t]{2}{*}{ Variables and categories } & \multirow{2}{*}{$\begin{array}{l}\text { Total } \\
\text { n (\%) }\end{array}$} & \multirow{2}{*}{$\begin{array}{l}\text { With spouse } \\
\mathrm{n}(\%)\end{array}$} & \multirow{2}{*}{$\begin{array}{l}\text { With non-spousal cohabitants } \\
\text { n (\%) }\end{array}$} & \multirow{2}{*}{$\begin{array}{l}\text { Living alone } \\
\mathrm{n}(\%)\end{array}$} & \multirow[b]{2}{*}{$p^{a}$} \\
\hline & & & & & \\
\hline \multicolumn{6}{|l|}{ Living arrangements } \\
\hline Living with spouse & $5645(85.5)$ & - & - & - & - \\
\hline Living with non-spousal cohabitants & $677(10.3)$ & - & - & - & - \\
\hline Living alone & $278(4.2)$ & - & - & - & - \\
\hline \multicolumn{6}{|l|}{ Age } \\
\hline $65-69$ & 2567 (38.9) & $2308(40.9)$ & $173(25.6)$ & $86(30.9)$ & \multirow[t]{5}{*}{$p<.001$} \\
\hline $70-74$ & $2044(31.0)$ & $1767(31.3)$ & $191(28.2)$ & $86(30.9)$ & \\
\hline $75-79$ & $1275(19.3)$ & $1048(18.6)$ & $159(23.5)$ & $68(24.5)$ & \\
\hline $80-84$ & $526(8.0)$ & $409(7.2)$ & $94(13.9)$ & $23(8.3)$ & \\
\hline 85 and older & $188(2.8)$ & $113(2.0)$ & $60(8.9)$ & $15(5.4)$ & \\
\hline \multicolumn{6}{|l|}{ Years of education } \\
\hline$<10$ & $3603(54.6)$ & $2991(53.0)$ & $458(67.7)$ & $154(55.4)$ & \multirow[t]{3}{*}{$p<.001$} \\
\hline$\geq 10$ & $2950(44.7)$ & $2618(46.4)$ & $211(31.2)$ & $121(43.5)$ & \\
\hline Missing & $47(0.7)$ & $36(0.6)$ & $8(1.2)$ & $3(1.1)$ & \\
\hline \multicolumn{6}{|l|}{ Household income } \\
\hline Low & $2263(34.3)$ & $1981(35.1)$ & $204(30.1)$ & $78(28.1)$ & \multirow[t]{4}{*}{$p<.001$} \\
\hline Middle & $3022(45.8)$ & $2633(46.6)$ & $267(39.4)$ & $122(43.9)$ & \\
\hline High & $731(11.1)$ & $616(10.9)$ & $95(14.0)$ & $20(7.2)$ & \\
\hline Missing & $54(8.8)$ & $415(7.4)$ & $111(16.4)$ & $58(20.9)$ & \\
\hline \multicolumn{6}{|l|}{ Self-rated health } \\
\hline Excellent/good & 4879 (73.9) & $4229(74.9)$ & $453(66.9)$ & $197(70.9)$ & \multirow[t]{3}{*}{$p<.001$} \\
\hline Fair/poor & $1659(25.1)$ & $1370(24.3)$ & $211(31.2)$ & $78(28.1)$ & \\
\hline Missing & $62(0.9)$ & $46(0.8)$ & $13(1.9)$ & $3(1.1)$ & \\
\hline \multicolumn{6}{|l|}{ Presence of illness } \\
\hline No & $1204(18.2)$ & $1036(18.4)$ & $115(17.0)$ & $53(19.1)$ & \multirow[t]{3}{*}{$p=.239$} \\
\hline Yes & $5181(78.5)$ & $4436(78.6)$ & $531(78.4)$ & $214(77.0)$ & \\
\hline Missing & $215(3.3)$ & $173(3.1)$ & $31(4.6)$ & $11(4.0)$ & \\
\hline \multicolumn{6}{|l|}{ Geriatric Depression Scale } \\
\hline No depression & $4304(65.2)$ & $3784(67.0)$ & $398(58.8)$ & $122(43.9)$ & \multirow[t]{4}{*}{$p<.001$} \\
\hline Depressive tendency & $1264(19.2)$ & $1037(18.4)$ & $153(22.6)$ & $74(26.6)$ & \\
\hline Depression & $364(5.5)$ & $273(4.8)$ & $51(7.5)$ & $40(14.4)$ & \\
\hline Missing & $668(10.1)$ & $551(9.8)$ & $75(11.1)$ & $42(15.1)$ & \\
\hline \multicolumn{6}{|l|}{ Instrumental activities of daily living } \\
\hline Without difficulty & 4935(74.8) & $4212(74.7)$ & $483(71.3)$ & $238(85.6)$ & \multirow[t]{3}{*}{$p<.001$} \\
\hline With difficulty & $1455(22.0)$ & $1256(22.2)$ & $165(23.8)$ & $34(12.2)$ & \\
\hline Missing & $210(3.2)$ & $175(3.0)$ & $29(4.3)$ & $6(2.2)$ & \\
\hline \multicolumn{6}{|l|}{ Subjective cognitive complaints } \\
\hline No & $5597(84.8)$ & $4811(85.2)$ & $549(81.1)$ & $237(85.3)$ & \multirow[t]{3}{*}{$p=.023$} \\
\hline Yes & $891(13.5)$ & $737(13.1)$ & $114(16.8)$ & $40(14.4)$ & \\
\hline Missing & $112(1.7)$ & $97(1.7)$ & $14(2.1)$ & $1(0.4)$ & \\
\hline
\end{tabular}


Table 1 Respondent characteristics by living arrangements for men $(N=6600)$ (Continued)

\begin{tabular}{|c|c|c|c|c|c|}
\hline \multicolumn{6}{|c|}{ Body mass index } \\
\hline$<18.5$ & $435(6.6)$ & $337(6.0)$ & $71(10.5)$ & $27(9.7)$ & \multirow[t]{4}{*}{$p<.001$} \\
\hline $18.5-24.9$ & $4685(71.0)$ & $4036(71.5)$ & $454(67.1)$ & $195(70.1)$ & \\
\hline$\geq 25$ & $1320(20.0)$ & $1147(20.3)$ & $125(18.5)$ & $48(17.3)$ & \\
\hline Missing & $160(2.4)$ & $125(2.2)$ & $27(4.0)$ & $8(2.9)$ & \\
\hline \multicolumn{6}{|c|}{ Alcohol consumption } \\
\hline No & $2700(40.9)$ & $2258(40.0)$ & $317(46.8)$ & $125(45.0)$ & \multirow[t]{3}{*}{$p=.007$} \\
\hline Yes & $3812(57.8)$ & $3312(58.7)$ & $350(51.7)$ & $150(54.0)$ & \\
\hline Missing & $88(1.3)$ & $75(1.3)$ & $10(1.5)$ & $3(1.1)$ & \\
\hline \multicolumn{6}{|l|}{ Smoking habit } \\
\hline None & $1737(26.3)$ & $1494(26.5)$ & $174(25.7)$ & $69(24.8)$ & \multirow[t]{3}{*}{$p=.073$} \\
\hline Past/current & 4657 (70.6) & 3989 (70.7) & $473(69.9)$ & $195(70.1)$ & \\
\hline Missing & $206(3.1)$ & $162(2.9)$ & $30(4.4)$ & $14(5.0)$ & \\
\hline \multicolumn{6}{|c|}{ Daily walking time } \\
\hline$\geq 30 \mathrm{~min}$ & $4105(62.2)$ & $3556(630)$ & $383(56.6)$ & $166(59.7)$ & \multirow[t]{3}{*}{$p=.015$} \\
\hline$<30 \min$ & 2069 (31.3) & 1735 (30.7) & $239(35.3)$ & $95(34.2)$ & \\
\hline Missing & $426(6.5)$ & $354(6.3)$ & $55(8.1)$ & $17(6.1)$ & \\
\hline \multicolumn{6}{|c|}{ Emotional support received } \\
\hline Yes & $5471(82.9)$ & $4776(84.6)$ & $505(74.6)$ & $190(68.3)$ & \multirow[t]{3}{*}{$p<.001$} \\
\hline No & $858(13.0)$ & $657(11.6)$ & $126(18.6)$ & $75(27.0)$ & \\
\hline Missing & $271(4.1)$ & $212(3.8)$ & $46(6.8)$ & $13(4.7)$ & \\
\hline \multicolumn{6}{|c|}{ Emotional support provided } \\
\hline Yes & $5179(78.5)$ & $4532(80.3)$ & $469(69.3)$ & $178(64.0)$ & \multirow[t]{3}{*}{$p<.001$} \\
\hline No & $1113(16.9)$ & $866(15.3)$ & $163(24.1)$ & $84(30.2)$ & \\
\hline Missing & $308(4.7)$ & $247(4.4)$ & $45(6.6)$ & $16(5.8)$ & \\
\hline \multicolumn{6}{|c|}{ Instrumental support received } \\
\hline Yes & $6157(933)$ & $5369(95.1)$ & $610(90.1)$ & $178(64.0)$ & \multirow[t]{3}{*}{$p<.001$} \\
\hline No & 239 (3.6) & $110(1.9)$ & $41(6.1)$ & $88(31.7)$ & \\
\hline Missing & $204(3.1)$ & $166(2.9)$ & $26(3.8)$ & $12(4.3)$ & \\
\hline \multicolumn{6}{|c|}{ Instrumental support provided } \\
\hline Yes & $5904(89.5)$ & $5221(92.5)$ & $518(76.5)$ & $165(59.4)$ & \multirow[t]{3}{*}{$p<.001$} \\
\hline No & $435(6.6)$ & $223(4.0)$ & $116(17.1)$ & $96(34.5)$ & \\
\hline Missing & $261(4.0)$ & $201(3.6)$ & $43(6.4)$ & $17(6.1)$ & \\
\hline
\end{tabular}

${ }^{\mathrm{a}} \mathrm{A}$ chi-square test was used to examine differences among living arrangement categories

cohabitants; $\mathrm{HR}=1.42$ for men living alone). When controlling for age, socioeconomic variables, and health variables, HR was 1.38 for men with non-spousal cohabitants, and was slightly increased $(\mathrm{HR}=1.45)$ for those living alone (Model 2). In women, both non-spouse household groups showed no significant relationship with BADL disability in Model 1, and living alone showed a significant relationship in Model 2 (HR =1.19). Among such differences in the relationship between living arrangements and the onset of BADL disability by gender, the interaction effect of living only with nonspousal cohabitants by gender was marginally significant $(p=.080)$, indicating a tendency toward a stronger association in men. A sub-analysis to examine differences between the two non-spouse household groups showed no significant difference for men or women in any models. For instance, compared to those who lived only with non-spousal cohabitants, the HR of those living alone was 1.12 (95\% confidence interval: 0.82-1.51) and 1.06 (95\% confidence interval: $0.90-1.25$ ) in men and women, respectively, controlling for age, socioeconomic status, and health variables.

The next three models revealed a substantial excess risk reduction for both non-spouse household groups in 
Table 2 Respondent characteristics by living arrangements for women ( $N=6868$ )

\begin{tabular}{|c|c|c|c|c|c|}
\hline Variables and categories & $\begin{array}{l}\text { Total } \\
\text { n (\%) }\end{array}$ & $\begin{array}{l}\text { With spouse } \\
\mathrm{n}(\%)\end{array}$ & $\begin{array}{l}\text { With non-spousal cohabitants } \\
\text { n (\%) }\end{array}$ & $\begin{array}{l}\text { Living alone } \\
\mathrm{n}(\%)\end{array}$ & $p^{\mathrm{a}}$ \\
\hline \multicolumn{6}{|l|}{ Living arrangements } \\
\hline Living with spouse & $3716(54.1)$ & - & - & - & - \\
\hline Living with non-spousal cohabitants & $2076(30.2)$ & - & - & - & - \\
\hline Living alone & $1076(15.7)$ & - & - & - & - \\
\hline \multicolumn{6}{|l|}{ Age } \\
\hline $65-69$ & $2417(35.2)$ & $1724(46.4)$ & $426(20.5)$ & $267(24.8)$ & \multirow[t]{5}{*}{$p<.001$} \\
\hline $70-74$ & $1993(29.0)$ & $1171(31.5)$ & $480(23.1)$ & $342(31.8)$ & \\
\hline $75-79$ & $1433(20.9)$ & $632(17.0)$ & $536(25.8)$ & $265(24.6)$ & \\
\hline $80-84$ & $701(10.2)$ & $166(4.5)$ & $388(18.7)$ & $147(13.7)$ & \\
\hline 85 and older & $324(4.7)$ & $23(0.6)$ & $246(11.8)$ & $55(5.1)$ & \\
\hline \multicolumn{6}{|l|}{ Years of education } \\
\hline$<10$ & $4294(62.5)$ & $2227(59.9)$ & $1430(68.9)$ & $637(59.2)$ & \multirow[t]{3}{*}{$p<.001$} \\
\hline$\geq 10$ & $2495(36.3)$ & $1460(39.3)$ & $614(29.6)$ & $421(39.1)$ & \\
\hline Missing & $79(1.2)$ & $29(0.8)$ & $32(1.5)$ & $18(1.7)$ & \\
\hline \multicolumn{6}{|l|}{ Household income } \\
\hline Low & $2257(32.9)$ & $1276(34.3)$ & $539(26.0)$ & $442(41.1)$ & \multirow[t]{4}{*}{$p<.001$} \\
\hline Middle & $2269(33.0)$ & $1498(40.3)$ & $545(26.3)$ & $226(21.0)$ & \\
\hline High & $637(9.3)$ & $330(8.9)$ & $279(13.4)$ & $28(2.6)$ & \\
\hline Missing & $1705(24.8)$ & $612(16.5)$ & $713(34.3)$ & $380(35.3)$ & \\
\hline \multicolumn{6}{|l|}{ Self-rated health } \\
\hline Excellent/good & $4994(72.7)$ & $2721(73.2)$ & $1493(71.9)$ & $780(72.5)$ & \multirow[t]{3}{*}{$p=.118$} \\
\hline Fair/poor & $1752(25.5)$ & $941(25.3)$ & $533(25.7)$ & $278(25.8)$ & \\
\hline Missing & $122(1.8)$ & $54(1.5)$ & $50(2.4)$ & $18(1.7)$ & \\
\hline \multicolumn{6}{|l|}{ Presence of illness } \\
\hline No & $1124(16.4)$ & $649(17.5)$ & $326(15.7)$ & $149(13.8)$ & \multirow[t]{3}{*}{$p=.013$} \\
\hline Yes & $5393(78.5)$ & $2896(77.9)$ & $1628(78.4)$ & $869(80.8)$ & \\
\hline Missing & $351(5.1)$ & $171(4.6)$ & $122(5.9)$ & $58(5.4)$ & \\
\hline \multicolumn{6}{|l|}{ Geriatric Depression Scale } \\
\hline No depression & $3961(57.7)$ & $2243(60.4)$ & $1180(56.8)$ & $538(50.0)$ & \multirow[t]{4}{*}{$p<.001$} \\
\hline Depressive tendency & 1319 (19.2) & 699 (18.8) & $376(18.1)$ & $244(22.7)$ & \\
\hline Depression & $384(5.6)$ & $187(5.0)$ & $128(6.2)$ & $69(6.4)$ & \\
\hline Missing & $1204(17.5)$ & $587(15.8)$ & $392(18.9)$ & $225(20.9)$ & \\
\hline \multicolumn{6}{|l|}{ Instrumental activities of daily living } \\
\hline Without difficulty & $5630(82.0)$ & $3229(86.9)$ & $1474(71.0)$ & $927(86.2)$ & \multirow[t]{3}{*}{$p<.001$} \\
\hline With difficulty & $1010(14.7)$ & $386(10.3)$ & $506(24.4)$ & $118(11.0)$ & \\
\hline Missing & $228(3.3)$ & $101(2.5)$ & $96(4.6)$ & $31(2.9)$ & \\
\hline \multicolumn{6}{|l|}{ Subjective cognitive complaints } \\
\hline No & $5673(82.6)$ & $3179(85.5)$ & $1601(77.1)$ & $893(83.0)$ & \multirow[t]{3}{*}{$p<.001$} \\
\hline Yes & $1035(15.1)$ & $464(12.5)$ & $414(19.9)$ & $157(14.6)$ & \\
\hline Missing & $160(2.3)$ & $73(1.9)$ & $61(2.9)$ & $26(2.4)$ & \\
\hline
\end{tabular}


Table 2 Respondent characteristics by living arrangements for women ( $N=6868$ ) (Continued)

\begin{tabular}{|c|c|c|c|c|c|}
\hline \multicolumn{6}{|c|}{ Body mass index } \\
\hline$<18.5$ & $531(7.7)$ & $245(6.6)$ & $190(9.2)$ & $96(8.9)$ & \multirow[t]{4}{*}{$p<.001$} \\
\hline $18.5-24.9$ & $4497(65.5)$ & $2498(67.2)$ & $1289(62.1)$ & $710(66.0)$ & \\
\hline$\geq 25$ & $1523(22.2)$ & $860(23.1)$ & $452(21.8)$ & $211(19.6)$ & \\
\hline Missing & $317(4.6)$ & $113(3.0)$ & $145(7.0)$ & $59(5.5)$ & \\
\hline \multicolumn{6}{|c|}{ Alcohol consumption } \\
\hline No & $5882(85.6)$ & 3157 (85.0) & $1818(87.6)$ & $907(84.3)$ & \multirow[t]{3}{*}{$p<.001$} \\
\hline Yes & $848(12.3)$ & $508(13.7)$ & $202(9.7)$ & $138(12.8)$ & \\
\hline Missing & $138(2.0)$ & $51(1.4)$ & $56(2.7)$ & $31(2.9)$ & \\
\hline \multicolumn{6}{|l|}{ Smoking habit } \\
\hline None & $6176(89.9)$ & 3387 (91.13) & $1845(88.9)$ & $944(87.7)$ & \multirow[t]{3}{*}{$p<.001$} \\
\hline Past/current & $411(6.0)$ & $206(5.5)$ & $122(5.9)$ & $83(7.7)$ & \\
\hline Missing & $281(4.1)$ & $123(3.3)$ & $109(5.3)$ & $49(4.6)$ & \\
\hline \multicolumn{6}{|c|}{ Daily walking time } \\
\hline$\geq 30 \mathrm{~min}$ & $3768(54.9)$ & $2078(55.9)$ & $1133(54.6)$ & $557(51.8)$ & \multirow[t]{3}{*}{$p=.015$} \\
\hline$<30 \min$ & 2187 (31.8) & $1147(30.9)$ & $651(31.4)$ & 389 (36.2) & \\
\hline Missing & $913(13.3)$ & $491(13.2)$ & $292(14.1)$ & $130(12.1)$ & \\
\hline \multicolumn{6}{|c|}{ Emotional support received } \\
\hline Yes & $6189(90.1)$ & 3402 (91.6) & $1856(89.4)$ & $931(86.5)$ & \multirow[t]{3}{*}{$p<.001$} \\
\hline No & $402(5.9)$ & $183(4.9)$ & $119(5.7)$ & $100(9.3)$ & \\
\hline Missing & $277(4.0)$ & $131(3.5)$ & $101(4.9)$ & $45(4.2)$ & \\
\hline \multicolumn{6}{|c|}{ Emotional support provided } \\
\hline Yes & $5587(81.3)$ & $3155(84.9)$ & $1566(75.4)$ & $866(80.5)$ & \multirow[t]{3}{*}{$p<.001$} \\
\hline No & $887(12.9)$ & $385(10.4)$ & $363(17.5)$ & $139(12.9)$ & \\
\hline Missing & $394(5.7)$ & $176(4.7)$ & $147(7.1)$ & $71(6.6)$ & \\
\hline \multicolumn{6}{|c|}{ Instrumental support received } \\
\hline Yes & $6119(89.1)$ & 3415 (91.9) & $1868(90.0)$ & $836(77.7)$ & \multirow[t]{3}{*}{$p<.001$} \\
\hline No & $502(7.3)$ & $180(4.8)$ & $125(6.0)$ & $197(18.3)$ & \\
\hline Missing & $247(3.6)$ & $121(3.3)$ & $83(4.0)$ & $43(4.0)$ & \\
\hline \multicolumn{6}{|c|}{ Instrumental support provided } \\
\hline Yes & $5996(87.3)$ & $3440(92.6)$ & $1732(83.4)$ & $824(76.6)$ & \multirow[t]{3}{*}{$p<.001$} \\
\hline No & $496(7.2)$ & $120(3.2)$ & $205(9.9)$ & $171(15.9)$ & \\
\hline Missing & $376(5.5)$ & $156(4.2)$ & $139(6.7)$ & $81(7.5)$ & \\
\hline
\end{tabular}

${ }^{\mathrm{a}} \mathrm{A}$ chi-square test was used to examine differences among living arrangement categories

men in Model 4, which controlled for social support variables within Model 2. The HR decreased from 1.45 (Model 2) to 1.34 (Model 4) in men living alone, indicating $24.4 \%$ excess risk reduction. For men living only with non-spousal cohabitants, a $15.8 \%$ excess risk reduction was found in Model 4 compared to Model 2. Additionally, to examine the relative importance of received or provided supports, we conducted an analysis entering the two support-received and two support-provided variables separately in Model 4, and found that the HR of men living alone was 1.40 in the model using only support-received variables, and 1.33 when controlling only for support provided variables. The same tendency was found in men who lived only with non-spousal cohabitants $(\mathrm{HR}=1.36$ and 1.32, respectively). As for women living alone, Model 4 showed a similar reduction in excess risk of BADL disability of $10.5 \%$ compared to Model 2, and social support provision variables almost explained the reduction. The mediation analysis revealed a significant mediating effect of social support in men, which represented $18 \%$ and $49 \%$ of the total effect in those living with non-spousal cohabitants $(p=.004)$ and those living alone $(p=.036)$, respectively. On 
Table 3 Incidence rate of basic activities of daily living disability onset during the follow-up period

\begin{tabular}{|c|c|c|c|c|c|c|c|}
\hline \multirow[t]{2}{*}{ Variables } & \multirow[t]{2}{*}{ Categories } & \multicolumn{3}{|l|}{ Men } & \multicolumn{3}{|l|}{ Women } \\
\hline & & $\begin{array}{l}\text { Incidence/ } \\
\text { person-year }\end{array}$ & $\begin{array}{l}\text { Incidence rate } \\
\text { per } 1000\end{array}$ & $p^{a}$ & $\begin{array}{l}\text { Incidence/ } \\
\text { person-year }\end{array}$ & $\begin{array}{l}\text { Incidence rate } \\
\text { per } 1000\end{array}$ & $p^{a}$ \\
\hline Total & - & $1108 / 51734$ & 21.4 & - & $1248 / 55850$ & 22.3 & - \\
\hline \multirow[t]{3}{*}{ Living arrangement } & Living with spouse & $871 / 44890$ & 19.4 & $p<.001$ & $473 / 31543$ & 15.0 & $p<.001$ \\
\hline & $\begin{array}{l}\text { Living with non-spousal } \\
\text { cohabitants }\end{array}$ & $171 / 4806$ & 35.6 & & $541 / 15897$ & 34.0 & \\
\hline & Living alone & $66 / 2039$ & 32.4 & & $234 / 8410$ & 27.8 & \\
\hline \multirow[t]{5}{*}{ Age } & $65-69$ & 184/21906 & 8.4 & $p<.001$ & $148 / 21341$ & 6.9 & $p<.001$ \\
\hline & $70-74$ & $309 / 16272$ & 19.0 & & $244 / 16573$ & 14.7 & \\
\hline & $75-79$ & $322 / 9216$ & 34.9 & & $357 / 11301$ & 31.6 & \\
\hline & $80-84$ & $199 / 3384$ & 58.8 & & $307 / 4955$ & 62.0 & \\
\hline & 85 and over & $94 / 955$ & 98.4 & & $192 / 1680$ & 114.3 & \\
\hline \multirow[t]{3}{*}{ Years of education } & $<10$ & $656 / 27872$ & 23.5 & $p<.001$ & $837 / 34714$ & 24.1 & $p<.001$ \\
\hline & $\geq 10$ & $441 / 23498$ & 18.8 & & $387 / 20572$ & 18.8 & \\
\hline & Missing & $11 / 364$ & 30.2 & & $24 / 565$ & 42.5 & \\
\hline \multirow[t]{4}{*}{ Equivalent income } & Low & $376 / 17,492$ & 21.5 & $p<.001$ & $407 / 18,495$ & 22.0 & $p<.001$ \\
\hline & Middle & $473 / 23995$ & 19.7 & & $310 / 18910$ & 16.4 & \\
\hline & High & $113 / 5910$ & 19.1 & & $119 / 5051$ & 23.6 & \\
\hline & Missing & $146 / 4336$ & 33.7 & & $412 / 13393$ & 30.8 & \\
\hline \multirow[t]{3}{*}{ Self-rated health } & Excellent/good & $704 / 39459$ & 17.8 & $p<.001$ & $749 / 41359$ & 18.1 & $p<.001$ \\
\hline & Fair/poor & $387 / 11844$ & 32.7 & & $469 / 13540$ & 34.6 & \\
\hline & Missing & $17 / 431$ & 39.4 & & $30 / 951$ & 31.6 & \\
\hline \multirow[t]{3}{*}{ Presence of illness } & No & 131/10138 & 12.9 & $p<.001$ & $120 / 9535$ & 12.6 & $p<.001$ \\
\hline & Yes & $941 / 39942$ & 23.6 & & $1060 / 43543$ & 24.3 & \\
\hline & Missing & $36 / 1655$ & 21.8 & & $68 / 2771$ & 24.5 & \\
\hline \multirow[t]{4}{*}{ Geriatric Depression Scale } & No depression & $619 / 34677$ & 17.9 & $p<.001$ & $583 / 32958$ & 17.7 & $p<.001$ \\
\hline & Depressive tendency & 250/9493 & 26.3 & & 289/10422 & 27.7 & \\
\hline & Depression & $89 / 2548$ & 34.9 & & $101 / 2941$ & 34.3 & \\
\hline & Missing & $150 / 5016$ & 29.9 & & $275 / 9529$ & 28.9 & \\
\hline \multirow[t]{3}{*}{ Instrumental activities of daily living } & Without difficulty & $724 / 39518$ & 18.3 & $p<.001$ & $778 / 47101$ & 16.5 & $p<.001$ \\
\hline & With difficulty & $324 / 10675$ & 30.4 & & $408 / 6996$ & 58.3 & \\
\hline & Missing & $60 / 1541$ & 38.9 & & $62 / 1753$ & 35.4 & \\
\hline \multirow[t]{3}{*}{ Subjective cognitive complaints } & No & $850 / 44512$ & 19.1 & $p<.001$ & $893 / 46860$ & 19.1 & $p<.001$ \\
\hline & Yes & $226 / 6428$ & 35.2 & & $307 / 7807$ & 39.3 & \\
\hline & Missing & $32 / 794$ & 40.3 & & $48 / 1183$ & 40.6 & \\
\hline \multirow[t]{4}{*}{ Body mass index } & $<18.5$ & $122 / 2938$ & 41.5 & $p<.001$ & $151 / 3920$ & 38.5 & $p<.001$ \\
\hline & $18.5-24.9$ & $767 / 37038$ & 20.7 & & $732 / 36996$ & 19.8 & \\
\hline & $\geq 25$ & $177 / 10653$ & 16.6 & & $240 / 12722$ & 18.9 & \\
\hline & Missing & $42 / 1105$ & 38.0 & & $125 / 2212$ & 56.5 & \\
\hline \multirow[t]{3}{*}{ Alcohol consumption } & No & $512 / 20451$ & 25.0 & $p<.001$ & $1105 / 47731$ & 23.2 & $p<.001$ \\
\hline & Yes & $577 / 30608$ & 18.9 & & $97 / 7109$ & 13.6 & \\
\hline & Missing & $19 / 675$ & 28.1 & & 46/1010 & 45.5 & \\
\hline
\end{tabular}


Table 3 Incidence rate of basic activities of daily living disability onset during the follow-up period (Continued)

\begin{tabular}{|c|c|c|c|c|c|c|c|}
\hline \multirow[t]{3}{*}{ Smoking habit } & None & 299/13849 & 21.6 & $p=.887$ & 1083/50509 & 21.4 & $p<.001$ \\
\hline & Past/current & $774 / 36350$ & 21.3 & & $82 / 3186$ & 25.7 & \\
\hline & Missing & $35 / 1535$ & 22.8 & & $83 / 2155$ & 38.5 & \\
\hline \multirow[t]{3}{*}{ Daily walking time } & $\geq 30 \mathrm{~min}$ & $632 / 32851$ & 19.2 & $p<.001$ & 637/30997 & 20.6 & $p<.001$ \\
\hline & $<30 \min$ & $411 / 15574$ & 26.4 & & $451 / 17498$ & 25.8 & \\
\hline & Missing & $65 / 3309$ & 19.6 & & $160 / 7355$ & 21.8 & \\
\hline \multirow[t]{3}{*}{ Emotional support received } & Yes & $851 / 43165$ & 19.7 & $p<.001$ & $1085 / 50517$ & 21.5 & $p<.001$ \\
\hline & No & $183 / 6589$ & 27.8 & & $92 / 3151$ & 29.2 & \\
\hline & Missing & $74 / 1980$ & 37.4 & & $71 / 2182$ & 32.5 & \\
\hline \multirow[t]{3}{*}{ Emotional support provided } & Yes & $781 / 41271$ & 18.9 & $p<.001$ & $916 / 46023$ & 19.9 & $p<.001$ \\
\hline & No & $240 / 8182$ & 29.3 & & $236 / 6740$ & 35.0 & \\
\hline & Missing & $87 / 2280$ & 38.2 & & $96 / 3086$ & 31.1 & \\
\hline \multirow[t]{3}{*}{ Instrumental support received } & Yes & $997 / 48425$ & 20.6 & $p<.001$ & 1090/49835 & 21.9 & $p=.031$ \\
\hline & No & $51 / 1812$ & 28.2 & & $100 / 4077$ & 24.5 & \\
\hline & Missing & $60 / 1498$ & 40.1 & & $58 / 1938$ & 29.9 & \\
\hline \multirow[t]{3}{*}{ Instrumental support provided } & Yes & $927 / 46692$ & 19.9 & $p<.001$ & $972 / 49306$ & 19.7 & $p<.001$ \\
\hline & No & $113 / 3101$ & 36.4 & & $164 / 3654$ & 44.9 & \\
\hline & Missing & $68 / 1940$ & 35.0 & & $112 / 2890$ & 38.8 & \\
\hline
\end{tabular}

${ }^{\mathrm{a} A}$ log-rank test was used to examine differences among categories for each study variable

the other hand, mediation effects of health-related variables and those of social support variables in women were nonsignificant [see Additional file 1: Table S1].

Finally, we conducted a series of sensitivity analyses. First, we limited respondents to those whose IADL were independent at baseline $(n=10,892)$ to avoid reverse causation in which mild disability affected living arrangements. The findings showed that HR for men living with non-spousal cohabitants and those living alone decreased from 1.38 and 1.45 to 1.28 and 1.36 , respectively, while HR for women living alone slightly increased to 1.28. Second, we excluded respondents who experienced spousal bereavement within a year $(n=537)$ to avoid the influence of this type of recent stressful life event on BADL disability. The findings showed that HR for men living with non-spousal cohabitants and those living alone were 1.33 and 1.41, respectively, while HR in women living alone decreased from 1.19 to 1.12.

\section{Discussion}

Under the premise of a relatively low rate of needs-driven cohabitation, this study showed a relationship between living arrangements and BADL disability onset, taking into consideration gender differences and cohabitation status of those living without a spouse. Indeed, in our data, only 429 among 13,468 cases (3.2\%) moved out during the 9.4 years of the follow-up period. The findings of this study showed that men living without a spouse, regardless of the presence of cohabitants, were more likely to develop BADL disability than those who lived with a spouse, while for women, a significant relationship was found only when they lived alone. Although the interaction effect of living only with non-spousal cohabitants by gender was only marginally significant, this study suggests higher risk in older men compared to women when they live only with non-spousal cohabitants. Several studies have also shown higher risk for BADL disability in older adults living only with non-spousal cohabitants compared to those living with a spouse $[12,15]$. However, our study provided new findings that excess risk of living with non-spousal cohabitants in older adults could depend on gender. Our findings suggest that men rely on spousal relationships for protecting their functional health, and that non-spousal cohabitants such as adult children do not compensate sufficiently for the role of the spouse. On the other hand, women may gain health protection from cohabitants, regardless of spousal relationships. Thus, the findings of this study confirmed the need to examine gender-specific risk assessments in the association between social relationships and functional health.

Contrary to previous studies $[11,13,16,17]$ showing a health advantage in older adults living alone compared to those living only with non-spousal cohabitants, the subgroup analysis in this study showed no significant differences in men and women despite using a larger or comparable sample size. This discrepancy might reflect a lower level of reverse causation in this study compared to previous studies, in which decreasing functional ability among older adults living alone leads to cohabitation with non-spousal family members such as adult children. 
Table 4 Risk of living arrangements on the onset of basic activities of daily living disability

\begin{tabular}{|c|c|c|}
\hline & Men & Women \\
\hline & $\operatorname{HR}(95 \% \mathrm{Cl})^{\mathrm{a}}$ & $\operatorname{HR}(95 \% \mathrm{Cl})^{\mathrm{a}}$ \\
\hline \multicolumn{3}{|l|}{ Model $1^{b}$} \\
\hline \multicolumn{3}{|l|}{ Living arrangements } \\
\hline $\begin{array}{l}\text { Living with spouse } \\
\text { (Reference) }\end{array}$ & 1.00 & 1.00 \\
\hline $\begin{array}{l}\text { Living with non-spousal } \\
\text { cohabitants }\end{array}$ & $1.39(1.18-1.64)$ & $1.09(0.95-1.26)$ \\
\hline Living alone & $1.42(1.11-1.83)$ & $1.14(0.97-1.35)$ \\
\hline \multicolumn{3}{|l|}{ Model $2^{c}$} \\
\hline \multicolumn{3}{|l|}{ Living arrangements } \\
\hline $\begin{array}{l}\text { Living with spouse } \\
\text { (Reference) }\end{array}$ & 1.00 & 1.00 \\
\hline $\begin{array}{l}\text { Living with non-spousal } \\
\text { cohabitants }\end{array}$ & $1.38(1.16-1.63)$ & $1.08(0.94-1.24)$ \\
\hline Living alone & $1.45(1.12-1.87)$ & $1.19(1.01-1.40)$ \\
\hline \multicolumn{3}{|l|}{ Model $3^{d}$} \\
\hline \multicolumn{3}{|l|}{ Living arrangements } \\
\hline $\begin{array}{l}\text { Living with spouse } \\
\text { (Reference) }\end{array}$ & 1.00 & 1.00 \\
\hline $\begin{array}{l}\text { Living with non-spousal } \\
\text { cohabitants }\end{array}$ & $1.37(1.16-1.62)$ & $1.09(0.95-1.25)$ \\
\hline Living alone & $1.45(1.12-1.87)$ & $1.19(1.01-1.40)$ \\
\hline \multicolumn{3}{|l|}{ Model $4^{\mathrm{e}}$} \\
\hline \multicolumn{3}{|l|}{ Living arrangements } \\
\hline $\begin{array}{l}\text { Living with spouse } \\
\text { (Reference) }\end{array}$ & 1.00 & 1.00 \\
\hline $\begin{array}{l}\text { Living with non-spousal } \\
\text { cohabitants }\end{array}$ & $1.32(1.11-1.57)$ & $1.07(0.93-1.23)$ \\
\hline Living alone & $1.34(1.02-1.76)$ & $1.17(0.98-1.38)$ \\
\hline \multicolumn{3}{|l|}{ Model $5^{f}$} \\
\hline \multicolumn{3}{|l|}{ Living arrangements } \\
\hline $\begin{array}{l}\text { Living with spouse } \\
\text { (Reference) }\end{array}$ & 1.00 & 1.00 \\
\hline $\begin{array}{l}\text { Living with non-spousal } \\
\text { cohabitants }\end{array}$ & $1.31(1.10-1.56)$ & $1.08(0.94-1.24)$ \\
\hline Living alone & $1.35(1.03-1.77)$ & $1.16(0.98-1.38)$ \\
\hline
\end{tabular}

${ }^{\mathrm{a}} \mathrm{HR}$ hazard ratio, $\mathrm{Cl}$ confidence interval

${ }^{b}$ Model 1: The effect of living arrangements on the outcome variable controlling for age

'Model 2: Model 1 + education + household income + health variables (self-rated health, presence of illness, depression, instrumental activities of daily living, and subjective cognitive complaints)

${ }^{d}$ Model 3: Model 2 + heath-related behavior variables (body mass index, alcohol consumption, smoking habits, and daily walking time) were controlled

e Model 4: Model 2 + social support variables (emotional support received, emotional support provided, instrumental support received, and instrumental support provided) were controlled

fModel 5: All covariates were controlled

Our findings also showed a potential pathway between living arrangements and BADL disability. A series of hierarchical analyses (Table 4) showed that social support exchange variables explained more excess risk than did health-related behaviors in men living alone and those living only with non-spousal cohabitants, with reduction rates of $24.4 \%$ and $15.8 \%$, respectively. In addition, a subsequent analysis showed the relative importance of support provision rather than support receipt by older adults in decreasing excess risk of BADL disability, even when controlling for predictors such as health. Social support provision is known to improve health in older adults $[40,41]$. Older men provide support mostly to their wife, while women provide support to more extended network members such as a spouse, children, or others [22]. Therefore, our findings suggest that living without a spouse could affect disability onset, partially due to lack of opportunities for support provision particularly in men. However, further research is necessary to examine the effect of social support provision and its pathways to functional disability, as the studies in this area are few [42].

Although the lack of social support exchange could explain part of the excess risk for BADL disability, men without spouses still had a significantly higher BADL disability risk than those living with a spouse after controlling for all covariates including mediator variables. This implies that the excess risk of BADL disability in men could be explained by unmeasured factors such as a decrease in social roles or self-efficacy [43].

\section{Limitations}

There were several limitations to this study. First, the onset of BADL was assessed based on the LTCI system; therefore, older adults with functional difficulty who had not applied for the LTCI benefit could have been misclassified as having no functional disabilities. However, such misclassification is less likely for the more severe levels of disability examined in this study [44]. Furthermore, BADL disability in this study was assessed using uniform nationwide criteria based on both a home-visit interview by a trained healthcare professional and a primary physician's opinion, suggesting that the outcome was less likely to suffer from self-report bias. However, further studies should replicate the findings of this study, using cut-off points reflecting more severe disability for LTCI care-needs or other BADL assessments.

Second, we measured living arrangements as well as covariates (for instance, IADL) only at baseline, which could have changed during the follow-up period of almost 10 years. Although the residential mobility rate in the data we used was very low during the follow-up period, implying less residential moves for support needs, it is still possible that respondents who had lived alone and then started to live with others during the follow-up period may have confounded the findings. In addition, more respondents could have experienced widowhood in the 
follow-up period, which may have led to an underestimation of the difference between those living with and without spouses in this study. Although we confirmed the excess risk of men living with non-spousal cohabitants and men living alone, even excluding those having a recent spousal bereavement from the analysis, we should further consider the effect of change in living arrangements in older adults to better understand the relationships between living arrangements and health.

Third, generalizability of this study is limited due to a moderate response rate $(52.1 \%)$; however, the respondents were selected randomly or completely enumerated from 10 municipalities in Japan.

\section{Implications}

Despite these limitations, the findings of our study could provide effective suggestions for the prevention of functional disability in community-dwelling older adults. Focusing on gender differences is important with respect to the role of living arrangements in the prevention of functional disability. Furthermore, more focus should be placed on support needs of older men living without spouses but with non-spousal cohabitants, in addition to those living alone, since older people with any cohabitants tend to be overlooked within the formal support system despite being at risk of BADL decline.

Policy makers and professionals should enhance opportunities for support exchange, particularly support provision by older adults. Social participation is known to be effective for disability prevention [27], and provides opportunities for social support exchange among participants [43]. Therefore, encouraging social participation for older adults at risk for BADL disability due to lack of social support exchange could be helpful. However, since Japanese older men are less likely to participate in these community groups than women [32], it is important to explore the needs and preferences of older men. For instance, a group for walking [32] or manufacturing products [45] might be relatively more acceptable to older men.

Future studies should consider the effect of change in marital status or cohabitation in the analytical model, since studies have shown that these changes could have a negative impact on health [46]. Furthermore, more detailed attention needs to be paid to the role of social support exchanges with spouses in preventing functional disabilities. This may provide practical suggestions regarding possible preventive services for the increasing population of individuals without spouses in aging societies.

\section{Conclusions}

This study examined the relationship between living arrangements and BADL disability onset in communitydwelling older adults, taking into consideration gender differences and cohabitation status of those living without a spouse. The findings showed that older men without spouses were more likely to develop disability onset regardless of cohabitants, while a marginal difference was found only for women living alone, confirming the necessity for gender-specific risk assessments of the effect of social relationships on functional health. Our study also revealed a potential pathway of social support exchange between living arrangements and BADL disability onset, and particularly the role of support giving by older adults. Policy makers and professionals should enhance opportunities for support exchange, and in particular, support giving by older adults who are at risk of disability.

\section{Additional file}

Additional file 1: Table S1. Mediation effects of health-related behavior variables (Model 3) and social support variables (Model 4). (XLSX 11 kb)

\section{Abbreviations \\ AGES: Aichi Gerontological Evaluation Study; BADL: Basic activities of daily living; HR: Hazard ratio; JAGES: Japan Gerontological Evaluation Study; \\ LTCl: Long-Term Care Insurance system}

\section{Acknowledgements}

We thank all JAGES project members for collaborating in our work. We also appreciate the work of Drs. H. Hirai, Y. Inoue, and S. Jeong in preparing the longitudinal dataset. This study used data from the Aichi Gerontological Evaluation Study (AGES) conducted by the Center for Well-being and Society, Nihon Fukushi University, as one of their research projects.

\section{Funding}

This study was supported by MEXT-Supported Program for the Strategic Research Foundation at Private Universities (2009-2013); Grant-in-Aid for Scientific Research (KAKENHI) (23,243,070, 18,390,200, and 25,713,027); and Grant (24-17 and 27-18) from the National Center for Gerontology and Geriatrics (NCGG).

\section{Availability of data and materials}

Data are from the AGES study, a part of the JAGES project. All enquiries are to be addressed to the data management committee via e-mail:

dataadmin.ml@jages.net. All JAGES datasets have ethical or legal restrictions for public deposition due to inclusion of sensitive information from the human participants.

\section{Authors' contributions \\ All authors contributed to the conception and design of this study. Data collection was primarily conducted by KK. Analyses were performed by TS and JA, and supported by CM. TS prepared the initial manuscript and CM, $J A$, and KK significantly contributed to revising it. All authors read and approved the final manuscript.}

\section{Ethics approval and consent to participate}

This survey project was conducted under a comprehensive agreement between each insurer and our research project team. The insurers took charge of conducting the survey and researchers analyzed anonymous data provided by the insurers. The study protocol and informed consent procedure were approved by the Nihon Fukushi University Ethics Committee.

Consent for publication

Not applicable.

Competing interests

The authors declare that they have no competing interests. 


\section{Publisher's Note}

Springer Nature remains neutral with regard to jurisdictional claims in published maps and institutional affiliations.

\section{Author details \\ 'Department of Social Science, National Center for Geriatrics and International and Community Oral Health, Tohoku University Graduate Aichi 474-8511, Japan. \\ Received: 10 October 2016 Accepted: 7 August 2017 Published online: 16 August 2017} Gerontology, 7-430 Morioka-cho, Obu, Aichi 474-8511, Japan. ${ }^{2}$ Department of School of Dentistry, Seiryo-machi, Aoba-ku, Sendai, Miyagi 980-8575, JAPAN. ${ }^{3}$ Center for Preventive Medical Science, Chiba University, 1-8-1 Inohana, Chuo-ku, Chiba 260-8670, Japan. ${ }^{4}$ Department of Gerontological Evaluation, National Center for Geriatrics and Gerontology, 7-430 Morioka-cho, Obu,

\section{References}

1. Aida J, Kondo K, Kawachi I, Subramanian SV, Ichida Y, Hirai H, Kondo N, Osaka K, Sheiham A, Tsakos G, et al. Does social capital affect the incidence of functional disability in older Japanese? A prospective population-based cohort study. J Epidemiol Community Health. 2013;67(1):42-7.

2. Prince MJ, Wu F, Guo Y, Gutierrez Robledo LM, O'Donnell M, Sullivan R, Yusuf S. The burden of disease in older people and implications for health policy and practice. Lancet. 2015;385(9967):549-62.

3. United Nations. World population prospects: The 2015 revision. New York: United Nations; 2015.

4. Tomassini C, Glaser K, Wolf DA, Broese van Groenou MI, Grundy E. Living arrangements among older people: an overview of trends in Europe and the USA. Popul Trends. 2004;115:24-34.

5. National Institute of Aging and World Health Organization. Global health and aging. Bethesda: NIH publication no. 11-7737; 2011.

6. Kehusmaa S, Autti-Ramo I, Helenius H, Hinkka K, Valaste M, Rissanen P. Factors associated with the utilization and costs of health and social services in frail elderly patients. BMC Health Serv Res. 2012;12:204

7. Lin IF, Brown SL. Unmarried boomers confront old age: a national portrait. Gerontologist. 2012:52(2):153-65.

8. Goldman N, Korenman S, Weinstein R. Marital status and health among the elderly. Soc Sci Med. 1995;40(12):1717-30.

9. Manzoli L, Villari P, MP G, Boccia A. Marital status and mortality in the elderly: a systematic review and meta-analysis. Soc Sci Med. 2007;64(1):77-94.

10. Joutsenniemi K, Martelin T, Martikainen P, Pirkola S, Koskinen S. Living arrangements and mental health in Finland. J Epidemiol Community Health. 2006;60(6):468-75.

11. Li LW, Zhang J, Liang J. Health among the oldest-old in China: which living arrangements make a difference? Soc Sci Med. 2009;68(2):220-7.

12. Rist PM, Liu SY, Glymour MM. Families and disability onset: are spousal resources less important for individuals at high risk of dementia? Am J Geriatr Psychiatry. 2016;24(7):585-94.

13. Lund R, Nilsson CJ, Avlund K. Can the higher risk of disability onset among older people who live alone be alleviated by strong social relations? A longitudinal study of non-disabled men and women. Age Ageing. 2010; 39(3):319-26.

14. Michael YL, Berkman LF, Colditz GA, Kawachi I. Living arrangements, social integration, and change in functional health status. Am J Epidemiol. 2001; 153(2):123-31.

15. Saito E, Ueki S, Yasuda N, Yamazaki S, Yasumura S. Risk factors of functional disability among community-dwelling elderly people by household in Japan: a prospective cohort study. BMC Geriatr. 2014;14:93.

16. Spalter T, Brodsky J, Shnoor Y. Improvements and decline in the physical functioning of Israeli older adults. Gerontologist. 2014;54(6):919-29.

17. van den Brink CL, Tijhuis M, van den Bos GA, Giampaoli S, Kivinen P, Nissinen A, Kromhout D. Effect of widowhood on disability onset in elderly men from three European countries. J Am Geriatr Soc. 2004;52(3):353-8.

18. Noel-Miller C. Spousal loss, children, and the risk of nursing home admission. J Gerontol B Psychol Sci Soc Sci. 2010;65b(3):370-80.

19. Wang D, Zheng J, Kurosawa M, Inaba Y, Kato N. Changes in activities of daily living $(A D L)$ among elderly Chinese by marital status, living arrangement, and availability of healthcare over a 3-year period. Environ Health Prev Med. 2009; 14(2):128-41.
20. Nilsson CJ, Lund R, Avlund K. Cohabitation status and onset of disability among older Danes: is social participation a possible mediator? J Aging Health. 2008; 20(2):235-53.

21. Rendall MS, Weden MM, Favreault MM, Waldron $\mathrm{H}$. The protective effect of marriage for survival: a review and update. Demography. 2011; 48(2):481-506.

22. Antonucci TC, Akiyama $\mathrm{H}$. An examination of sex differences in social support among older men and women. Sex Roles. 1987;17(11):737-49.

23. Silverstein $M$, Angelelli JJ. Older parents' expectations of moving closer to their children. J Gerontol B Psychol Sci Soc Sci. 1998;53(3):S153-63.

24. Brown JW, Liang J, Krause N, Akiyama H, Sugisawa H, Fukaya T. Transitions in living arrangements among elders in Japan: does health make a difference? J Gerontol B Psychol Sci Soc Sci. 2002;57(4):S209-20.

25. Takagi E, Silverstein M, Crimmins E. Intergenerational coresidence of older adults in Japan: conditions for cultural plasticity. J Gerontol B Psychol Sci Soc Sci. 2007;62(5):S330-9.

26. Ministry of Health, Labour, and Welfare. Vital statistics http://www.mhlw.go. jp/toukei/list/81-1a.html. Accessed 27 Sep 2016.

27. Kanamori S, Kai Y, Aida J, Kondo K, Kawachi I, Hirai H, Shirai K, Ishikawa Y, Suzuki K, Group J. Social participation and the prevention of functional disability in older Japanese: the JAGES cohort study. PLoS One. 2014;9(6):e99638.

28. Tsutsui T, Muramatsu N. Care-needs certification in the long-term care insurance system of Japan. J Am Geriatr Soc. 2005;53(3):522-7.

29. Ministry of Health, Labour, and Welfare. Long-term care, health and welfare services for the elderly. http://www.mhlw.go.jp/english/policy/care-welfare/ care-welfare-elderly/index.html. Accessed 27 Sep 2016.

30. Saito O. Marriage, family labour and the stem family household: traditional Japan in a comparative perspective. Contin Chang. 2000;15(1):17-45.

31. Cabinet office of Japan: [Annual report on the aging society: 2016]. http:// www8.cao.go.jp/kourei/whitepaper/w-2016/html/zenbun/index.html. Accessed 22 Feb 2017.

32. Saito T, Kondo K, Murata C, Jeong S, Suzuki K, Kondo N, Group J. Gender and regional differences in going-out, social, and leisure activities among older adults. Findings from the JAGES Project. Nihon Koshu Eisei Zasshi. 2015;62(10):596-608

33. Wong CH, Wong SF, Pang WS, Azizah MY, Dass MJ. Habitual walking and its correlation to better physical function: implications for prevention of physical disability in older persons. J Gerontol A Biol Sci Med Sci. 2003; 58(6):555-60.

34. Stuck AE, Walthert JM, Nikolaus T, Bula CJ, Hohmann C, Beck JC. Risk factors for functional status decline in community-living elderly people: a systematic literature review. Soc Sci Med. 1999;48(4):445-69.

35. Yesavage JA, Brink TL, Rose TL, Lum O, Huang V, Adey M, Leirer VO. Development and validation of a geriatric depression screening scale: $a$ preliminary report. J Psychiatr Res. 1982;17(1):37-49.

36. Lawton MP. Assessing the competence of older people. In: Kent D, Kastenbaum R, Sherwood S, editors. Research Planning and Action for the Elderly: the Power and Potential of Social Science. New York: Behavioral Publications; 1972. p. 122-43.

37. Koyano W, Shibata H, Nakazato K, Haga H, Suyama Y. Measurement of competence: reliability and validity of the TMIG Index of Competence. Arch Gerontol Geriatr. 1991;13(2):103-16.

38. Buis ML. Direct and indirect effects in a logit model. Stata J. 2010;10(1):11-29.

39. Rubin DB. Multiple imputation after 18 years. J Am Stat Assoc. 1996; 91(434):473-89.

40. Brown SL, Nesse RM, Vinokur AD, Smith DM. Providing social support may be more beneficial than receiving it: results from a prospective study of mortality. Psychol Sci. 2003;14(4):320-7.

41. Thomas PA. Is it better to give or to receive? Social support and the wellbeing of older adults. J Gerontol Soc Sci. 2010;65B(3):351-7.

42. Inagaki TK, Bryne Haltom KE, Suzuki S, Jevtic I, Hornstein E, Bower JE, Eisenberger NI. The neurobiology of giving versus receiving support: the role of stress-related and social reward-related neural activity. Psychosom Med. 2016;78(4):443-53.

43. Berkman LF, Glass T, Brissette I, Seeman TE. From social integration to health: Durkheim in the new millennium. Soc Sci Med. 2000;51(6):843-57.

44. Fujiwara Y, Amano H, Kumagai S, Yoshida H, Fujita K, Naito T, Watanabe N, Nishi M, Mori S, Shinkai S. Physical and psychological predictors for the onset of certification of long-term care insurance among older adults living independently in a community a 40-month follow-up study. Nihon Koshu Eisei Zasshi. 2006;53(2):77-91. 
45. Okubo S, Saito T, Lee HJ, Yoshie S, Wakui K, Kai I. Correlates to male participation in disability prevention programs for the elderly. Nihon Koshu Eisei Zasshi. 2005: 52(12):1050-8.

46. Blomgren J, Martikainen P, Grundy E, Koskinen S. Marital history 1971-91 and mortality 1991-2004 in England \& Wales and Finland. J Epidemiol Community Health. 2012;66(1):30-6

Submit your next manuscript to BioMed Central and we will help you at every step:

- We accept pre-submission inquiries

- Our selector tool helps you to find the most relevant journal

- We provide round the clock customer support

- Convenient online submission

- Thorough peer review

- Inclusion in PubMed and all major indexing services

- Maximum visibility for your research

Submit your manuscript at www.biomedcentral.com/submit 